\title{
Unsupervised self-testing of airway obstruction by forced oscillation at the patient's home
}

\author{
J. Rigau*, F. Burgos", C. Hernández", J. Roca*, D. Navajas*, R. Farré*
}

Unsupervised self-testing of airway obstruction by forced oscillation at the patient's home. J. Rigau, F. Burgos, C. Hernández, J. Roca, D. Navajas, R. Farré. (C)ERS Journals Ltd 2003.

ABSTRACT: As active patient cooperation is not required, the forced oscillation technique (FOT) could be suitable for measuring airway obstruction in routine home applications. Nevertheless, FOT has never been used at the patient's home to date. The aim of this study was to assess the feasibility of FOT and the reproducibility of measured respiratory resistance $(R \mathrm{rs})$ in routine patient self-testing at home.

Altogether, nine asthmatic patients self-measured their $R$ rs with a portable FOT device and spirometry for 10-14 days, in the morning and evening, and before and after bronchodilator inhalation. During each measurement session, the patients carried out four consecutive FOT measurements. Grubbs' discordancy test for detecting outliers was used to evaluate intermeasure reliability.

Only $4.9 \%$ of $R$ rs data reported by patients were rejected as artefacts. The coefficient of variation of $R$ rs was $7.9 \pm 6.3 \%$ (mean \pm SD). When compared with spirometry, the per cent change in $R$ rs for detecting a positive bronchodilator response showed an $83 \%$ sensitivity and $72 \%$ specificity.

Unsupervised self-measurement of respiratory resistance at the patient's home provided results similar to those obtained by a technician in a lung function lab. Forced oscillation technique could be a useful tool for the routine follow-up of asthmatic patients at home.

Eur Respir J 2003; 22: 668-671.
*Unitat de Biofísica i Bioenginyeria, Facultat de Medicina, Universitat de Barcelona, and ${ }^{\#}$ Servei de Pneumologia, Hospital Clínic, Institut d'Investigacions Biomèdiques August Pi i Sunyer, Barcelona, Spain.

Correspondence: R. Farré, Unitat de Biofísica i Bioenginyeria, Facultat de Medicina, Casanova 143, 08036 Barcelona, Spain.

Fax: 34934024516

E-mail: rfarre@ub.edu

Keywords: Airway impedance, asthma, chronic obstructive pulmonary disease, home monitoring, respiratory resistance

Received: January 292003

Accepted after revision: April 232003

This work was supported in part by Ministerio de Ciencia y Tecnología (SAF2002-03616), Fondo Investigaciones Sanitarias (99-0034-03) and Red Respira C03/11 SEPAR-99 and the European Commission (CHRONIC, V Program, IST_DG XIII, 1999-12158).
The rising prevalence of chronic respiratory diseases and technological advances in medicine are leading to an increasing demand for health services at the patient's home [1]. Spirometry is the gold standard for assessing lung function in home care applications. However, since active patient cooperation is required, spirometric measurements in accordance with standardised criteria [2,3] are difficult to obtain in many paediatric and elderly patients [4]. Moreover, spirometric repeatability is difficult to achieve in asthmatic patients, given that the forced manoeuvres may alter the bronchomotor tone [5]. The difficulty in obtaining reproducible spirometric measurements in these subgroups of patients is increased when there is no technician to supervise the patient when self-testing their lung function at home. Therefore, a tool that is alternative or complementary to spirometry would be helpful for evaluating lung function during the follow-up of respiratory patients at home.

The forced oscillation technique (FOT) is useful for assessing the degree of airway obstruction in patients with chronic respiratory diseases [6-9]. FOT is based on the application of a small pressure oscillation $\left(1 \mathrm{cmH}_{2} \mathrm{O}\right)$ at the mouth during spontaneous breathing and on the measurement of the respiratory resistance (Rrs), i.e. the real part of the respiratory impedance, at a frequency higher than the breathing rate. Since minimal patient cooperation is required, FOT could be a suitable tool for the routine home monitoring of airway obstruction in asthmatic patients [9, 10]. This application is currently possible by using a recently designed portable FOT device [11]. Nevertheless, as FOT measurements are usually carried out by a technician in the lung function $\mathrm{lab}$, there are no data currently available on the feasibility of unsupervised FOT self-measurement at the patient's home. The aim of this pilot study was to assess the feasibility of FOT and the reproducibility of $R$ rs data in routine unsupervised home self-testing in asthmatic patients.

\section{Methods}

The study was carried out using nine patients, aged $44 \pm 9$ yrs (mean $\pm \mathrm{SD}$ ), including six males, with well characterised asthma (forced expiratory volume in one second (FEV1) $71 \pm 30 \%$ predicted; forced vital capacity $(\mathrm{FVC})=4.0 \pm$ $1.1 \mathrm{~L} ; \mathrm{FEV} 1 / \mathrm{FVC} 62 \pm 18 \%$ ) in a clinically stable condition. The protocol was approved by the Ethics Committee of the Hospital Clinic Provincial, Barcelona, and informed written consent was obtained from all patients. Patients were trained in FOT for 15 min and were provided with a portable FOT device [11] with written instructions and a diary card. Eight of the patients were also supplied with a portable spirometer (Datospir 70; Sibel SA, Barcelona, Spain).

The patient performed two daily measurement sessions at home: one in the morning and one in the evening (fig. 1). In each session, the patient checked the calibration of the portable FOT device by measuring a reference resistance incorporated into the device [11]. Subsequently, the patient self-measured $R$ rs, while holding their cheeks during spontaneous breathing and wearing a noseclip. Four consecutive FOT 


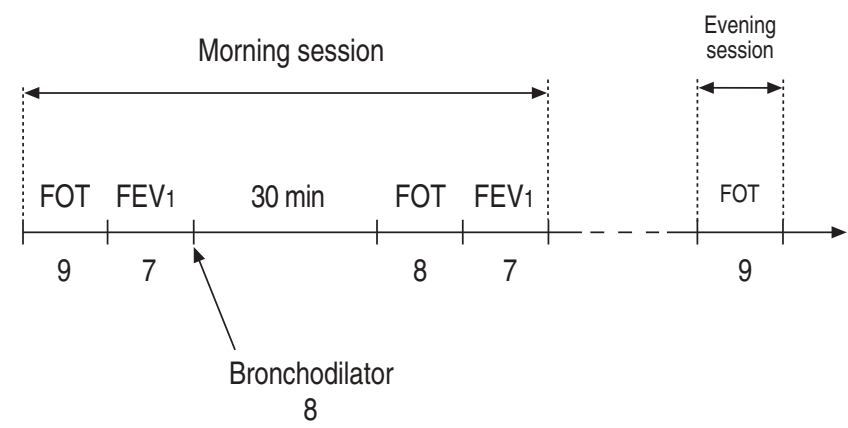

Fig. 1.-Diagram of the different tests performed by patients at home in the measurement protocol for each day of the study. The numbers indicate the number of subjects that reported data for each test. One patient did not perform spirometry. In another patient forced expiratory volume in one second $\left(\mathrm{FEV}_{1}\right)$ data were lost owing to a technical problem in the portable spirometer. FOT: forced oscillation technique.

measurements at a single frequency $(5 \mathrm{~Hz}, 16 \mathrm{~s}$ each) were carried out in each session. The FOT device computed the coherence function $\left(\gamma^{2}\right)$ for assessing intra-measure reliability [11]. In case of a measurement with $\gamma^{2}<0.95$, the $R$ rs data was automatically discarded and a red light in the front panel of the device indicated that the patient should repeat the measurement. In the morning session and after the FOT measurements, the patient performed three FEV1 measurements $[2,3]$. The portable spirometer stored the best of these three measurements in its internal memory for later analysis. Subsequently, the patient inhaled $300 \mu \mathrm{g}$ of salbutamol and after 30 min repeated the FOT and spirometric measurements.

The Rrs and FEV1 were analysed at the end of the home measurement period, which lasted 10-14 days depending on the day when the protocol started. When the calibration test exceeded a $\pm 5 \%$ tolerance range, the $R$ rs data corresponding to this session were rejected. To detect possible outliers, the Grubbs' discordancy test ( $\alpha=0.05$ significance level) [12], was applied to the four Rrs data in each measurement. The detected outliers were regarded as an artefact and rejected. The mean and the coefficient of variation (CV) of $R$ rs in each FOT measurement were computed from the accepted data. Changes in $R$ rs and in $\mathrm{CV}(R \mathrm{rs})$ between the morning prebronchodilator, morning postbronchodilator and evening measurements and between the different days of the study were evaluated with two-way repeated measurements analysis of variance (ANOVA) $(\mathrm{p}<0.05)$.

The changes in $R$ rs induced by the bronchodilator were analysed by means of two indices: $\Delta \% \operatorname{Rrs}$ and $\Delta R \mathrm{rs} / \mathrm{SD} . \Delta \% \operatorname{Rrs}$ is the per cent change from prebronchodilator $R \mathrm{rs}$, and $\Delta R \mathrm{rs} /$ $\mathrm{SD}$ is the quotient between the post and prebronchodilator $R$ rs difference $(\Delta R \mathrm{rs})$ and the SD of the prebronchodilator measurements. The sensitivity and the specificity of $\Delta \% R$ rs and $\Delta R \mathrm{rs} / \mathrm{SD}$ were examined using their corresponding receiveroperating characteristic (ROC) curve $[13,14]$, in accordance with the presence of significant bronchodilator response (FEV1 $\%$ change $\geqslant 12 \%$ and absolute change in FEV $1 \geqslant 200 \mathrm{~mL}$ ) [15]. The optimal cut-off value for $\Delta \% R$ rs and $\Delta R \mathrm{rs} / \mathrm{sD}$ was determined from the closest point to the upper left-hand corner of the ROC curve [13, 14].

\section{Results}

Rejection of Rrs data owing to a calibration value outside the tolerance range was necessary in only three of the 26 FOT sessions carried out by one of the patients. Two patients did not perform the measurements in one session. Only 30 of
1,215 Rrs data obtained in the sessions with acceptable calibration values were rejected according to the outliers test.

The $R$ rs measured in all patients during the whole study in the morning before and after bronchodilator were significantly different: $5.25 \pm 2.27 \mathrm{cmH}_{2} \mathrm{O} \cdot \mathrm{s} \cdot \mathrm{L}^{-1}$ and $4.82 \pm 2.46 \mathrm{cmH}_{2} \mathrm{O} \cdot \mathrm{s} \cdot \mathrm{L}^{-1}$, respectively. The evening $\operatorname{Rrs}\left(5.26 \pm 2.28 \mathrm{cmH}_{2} \mathrm{O} \cdot \mathrm{s} \cdot \mathrm{L}^{-1}\right)$ was not significantly different from the morning baseline value. No significant differences were found between the different days. Figure 2 shows the mean $( \pm \mathrm{SD})$ of the $\mathrm{CV}(R \mathrm{rs})$ for the pre and postbronchodilator measurements in the morning and the measurement in the evening corresponding to the first 10 days of the study. The ANOVA test showed no significant differences in the $\mathrm{CV}(\mathrm{Rrs})$ between the different measurements in each day and between the different days. On average, $\mathrm{CV}(\mathrm{Rrs})$ for all patients and measurements was $7.9 \pm 6.3 \%$.

From the total 76 measurement sessions after bronchodilator inhalation, 23 showed a significant reversibility in accordance with the conventional criteria based on FEV1. The ROC curves in figure 3 show the possible combinations
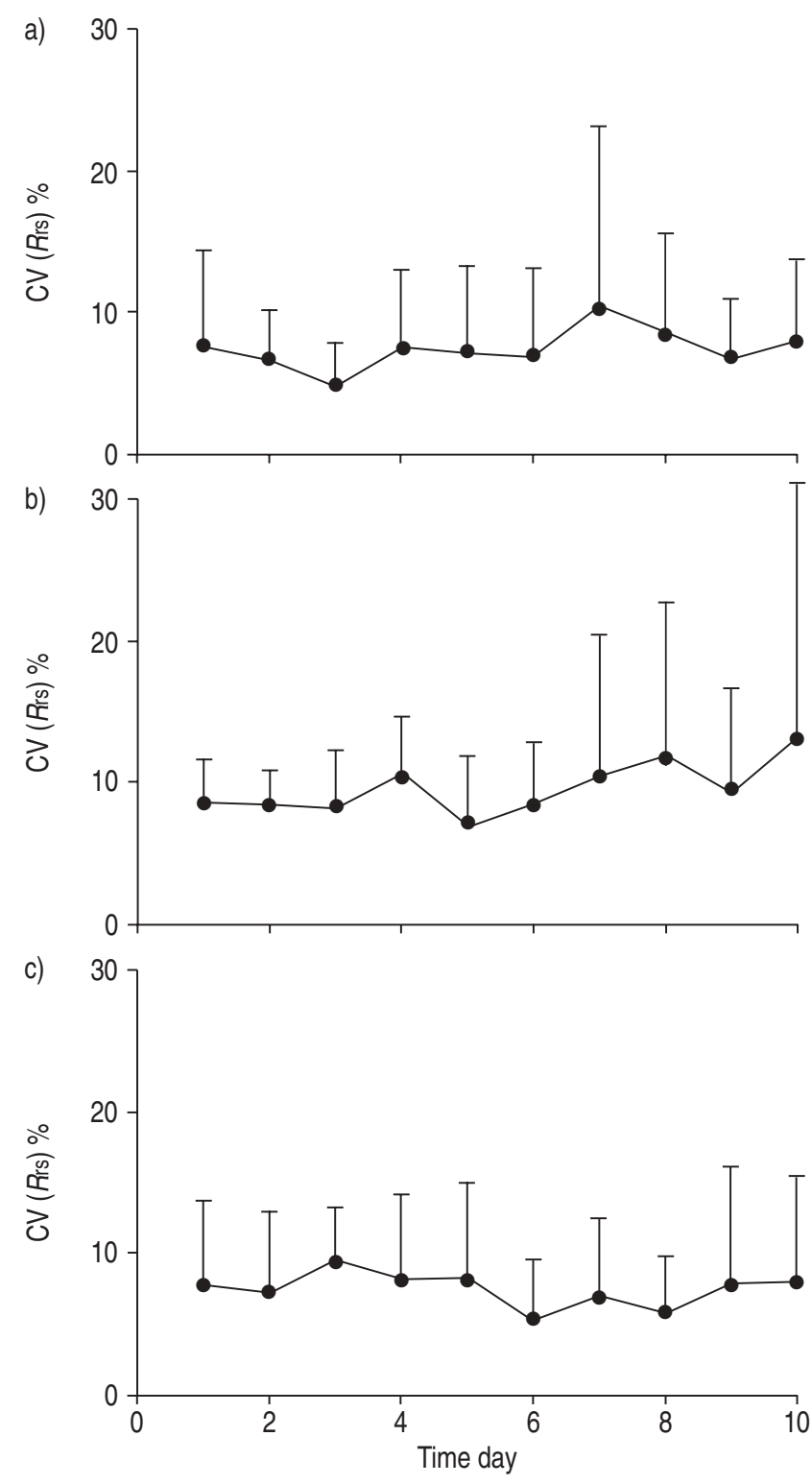

Fig. 2. - Coefficient of variation of respiratory resistance $(\mathrm{CV}(\mathrm{Rrs}))$ for a) morning baseline, b) morning postbronchodilator and c) evening sessions and days of the study. Data are presented as mean+SD. 


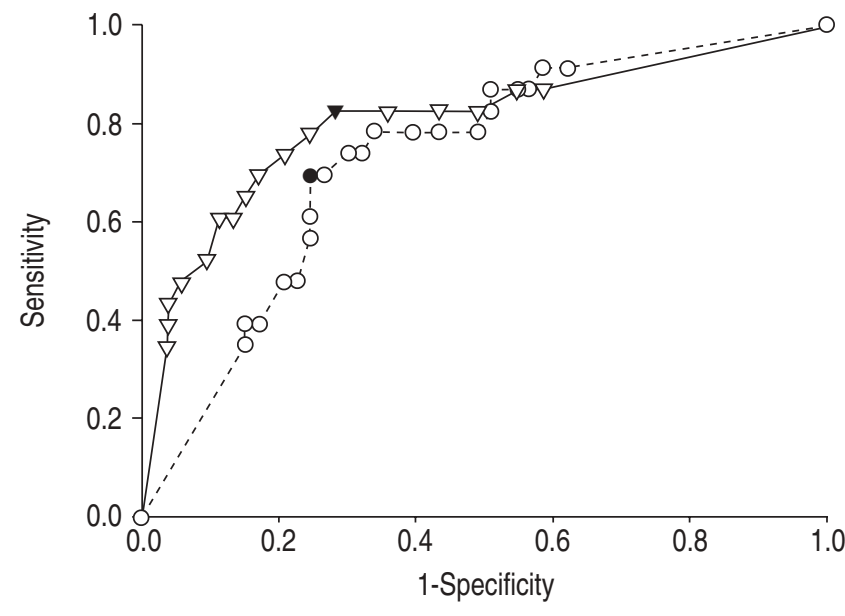

Fig. 3.-Receiver operating characteristic curves for two indices describing the change of respiratory resistance $(R \mathrm{rs})$ after the inhalation of $300 \mu \mathrm{g}$ of salbutamol. $\nabla$ : per cent change in Rrs from baseline; $\bigcirc$ : quotient between the post and prebronchodilator $R$ rs difference $(\Delta R \mathrm{rs})$ and the $\mathrm{SD}$ of the prebronchodilator measurements $(\Delta R \mathrm{rs} / \mathrm{SD}) ;$

between the true-positive ratio (sensitivity) and the falsepositive ratio (1-specificity) for the different thresholds defining positive response to salbutamol for the indices $\Delta \% R$ rs and $\Delta R \mathrm{rs} / \mathrm{SD}$. The best cut-off point for $\Delta \% R$ rs to discriminate between FOT measurements with a positive bronchodilator response and those with a negative response was a $13 \%$ decrease in Rrs. The sensitivity and the specificity at this cutoff value were $83 \%$ and $72 \%$, respectively. The best cut-off point for the $\Delta R$ rs/SD index was -2.8 , yielding $70 \%$ sensitivity and $76 \%$ specificity.

\section{Discussion}

This study showed that all the investigated patients were able to self-measure their $R$ rs at home without supervision. The amount of artefactual data was low $(4.9 \%)$ and the variability of unsupervised self-measurements $(\mathrm{CV}(\mathrm{Rrs})=7.9 \%)$ was similar to that reported in asthmatic adults from measurements performed by a technician in the lung function lab (ranging 4.9-10.3\%) [16, 17].

One important quality control issue in applications where the patients self-measure their lung function without the supervision of a technician is the periodic calibration of the measuring system. This procedure was implemented in the device used and was systematically carried out by the patients. Only $1.4 \%$ of the total FOT measurement sessions were rejected owing to a calibration test outside the tolerance range. As these sessions were immediately preceded and followed by sessions with correct calibration data, the artefact was probably due to an incorrect connection of the transducer head to the reference resistance, rather than to a malfunction of the FOT device. Another important quality control issue in home FOT measurements concerns the reliability of the measured Rrs. For each measurement, the portable FOT device computed $\gamma^{2}$ [11], which is a conventional procedure for assessing intra-measure reliability in FOT measurements [18]. From the quality control viewpoint, an important difference between FOT measurements carried out by a technician and those performed by the patient concerns the evaluation of the inter-measure reliability of the various $R$ rs values obtained in a given measuring session. The conventional procedure to detect and reject possible artefacts (swallowing, sighs, cough, etc.) is based on simple visual inspection of the raw pressure and flow signals by the technician. In home applications this quality control procedure could be implemented by including a telecommunication interface in the FOT device for signal transmission, as in other telemonitoring settings for respiratory patients $[19,20]$. Alternatively, in unsupervised measurements, these artefacts can be rejected by implementing a procedure to detect outliers.

The Grubbs' discordancy test used in this study is a conventional statistical method for detecting outliers [12]. Remarkably, this test is especially suitable for small samples, as in this work, where only four FOT measures were recorded in each session. Only $2.5 \%$ of the Rrs data with acceptable calibration were detected as outliers and rejected. Possible causes for these extreme values were an incorrect observance of the protocol during the measurement (noseclip wrongly placed, cheeks not held, mouth partially open, etc.), irregular breathing (swallowing, sighs, cough, etc.) or an error when recording the data on the diary card. To analyse the robustness of the outliers detection procedure, the authors repeated the data analysis by using Dixon's discordancy test, which is also suitable for small samples [12]. This test yielded practically the same results as Grubbs' test. Out of a total of 31 outliers detected by Dixon's discordancy test, 29 of them coincided with those detected by Grubbs' test.

In addition to assessing the feasibility of FOT and the reliability of $R$ rs in home care applications, the authors also evaluated the capability of FOT for detecting changes in airway obstruction at the patient's home. To this end, the sensitivity and the specificity of FOT for detecting a positive bronchodilator response were compared with the standardised criteria based on spirometry [15]. The per cent change in $R$ rs from baseline $(\Delta \% R \mathrm{rs})$ showed a sensitivity and a specificity similar to those reported in studies where FOT measurements were carried out in asthmatic children by a technician in a lung function lab $[21,22]$. The cut-off value for $\Delta \% \operatorname{Rrs}(13 \%)$ resembled the $10 \%$ change in respiratory conductance reported by ZERAH et al. [7] in adult asthmatic patients. However, the discriminative value in the current study was lower than others reported for asthmatic children [8, 21-23]. This could be explained by the studying of different populations and indices to quantify the bronchodilator response. The cut-off value of $\Delta R \mathrm{rs} / \mathrm{SD}$ indicated that an absolute decrease in $\operatorname{Rrs} 2.8$ times greater than the baseline SD was associated with a positive bronchodilator response. This cut-off value provided a sensitivity $(70 \%)$ and a specificity (76\%) close to those reported for quantifying changes in Rrs, normalised to measurement variance in asthmatic children (sensitivity and specificity ranging $69-79 \%$ and $65-78 \%$, respectively) [22-24].

In conclusion, the results of this pilot study demonstrate that, with simple patient training, unsupervised forced oscillation technique self-measurements at home are feasible and provide airway obstruction data with reliability similar to that reported from measurements carried out in the lung function lab. Despite extensive data demonstrating that forced oscillation technique is able to monitor airway obstruction, different information is provided by this technique and by spirometry, given that lung function is assessed under different conditions, i.e. during spontaneous breathing in forced oscillation technique and during a forced manoeuvre in spirometry. As spirometry is the reference tool for assessing lung function and as there are no conclusive data on the correspondence of the obstruction indices provided by both techniques, forced oscillation technique should be regarded as a complementary tool of spirometry in general patients. Nevertheless, in some subpopulations, namely children and elderly patients, where spirometry is difficult or impossible, forced oscillation technique could be an alternative 
technique for respiratory home follow-up in patients with obstructive diseases.

Acknowledgements. The authors wish to thank M.A. Rodriguez for his technical assistance and S.A. Sibel (Barcelona, Spain) for providing the portable spirometer.

\section{References}

1. Clini E, Vitacca M, Foglio K, Simoni P, Ambrosino N. Long-term home care programmes may reduce hospital admissions in COPD with chronic hypercapnia. Eur Respir $J$ 1996; 9: 1605-1610.

2. American Thoracic Society. Lung function testing: selection of reference values and interpretative strategies. Am Rev Respir Dis 1991; 144: 1202-1218.

3. European Respiratory Society. Standardized lung function testing. Lung volumes and forced ventilatory flows. 1993 update. Eur Respir J 1993; 6: 5-40.

4. Carvalhaes-Neto N, Lorino $\mathrm{H}$, Gallinari C, et al. Cognitive function and assessment of lung function in the elderly. Am J Respir Crit Care Med 1995; 152: 1611-1615.

5. Gayrard P, Orehek J, Grimaud C, Charpin J. Bronchoconstrictor effects of a deep inspiration in patients with asthma. Am Rev Respir Dis 1975; 111: 433-439.

6. Farré R, Peslin R, Rotger M, Barbera JA, Navajas D. Forced oscillation total respiratory resistance and spontaneous breathing lung resistance in COPD patients. Eur Respir J 1999; 14: 172-178.

7. Zerah F, Lorino AM, Lorino H, Harf A, Macquin-Mavier I. Forced oscillation technique vs spirometry to assess bronchodilatation in patients with asthma and COPD. Chest 1995; 108: 41-47.

8. Ducharme FM, Davis GM. Respiratory resistance in the emergency department. A reproducible and responsive measure of asthma severity. Chest 1998; 113: 1566-1572.

9. Farré R, Rotger M, Marchal F, Peslin R, Navajas D. Assessment of bronchial reactivity by forced oscillation admittance avoids the upper airway artefact. Eur Respir $J$ 1999; 13: 761-766.

10. Que C-L, Kenyon CM, Olivenstein R, Macklem PT, Maksym GN. Homeokinesis and short-term variability of human airway caliber. J Appl Physiol 2001; 91: 1131-1141.

11. Rigau J, Farré R, Roca J, Marco S, Herms A, Navajas D. A portable forced oscillation device for respiratory home monitoring. Eur Respir J 2002; 19: 146-150.

12. Barnett V, Lewis T. Outliers in statistical data. Ontario, John Wiley and Sons, 1984.

13. Robertson EA, Zweig MH. Use of receiver operating characteristic curves to evaluate the clinical performance of analytical systems. Clin Chem 1981; 27: 1569-1574.

14. Altman DG. Practical statistics for medical research. London, Chapman \& Hall, 1991.

15. American Thoracic Society. Lung function testing: selection of reference values and interpretative strategies. Am Rev Respir Dis 1991; 144: 1202-1218.

16. Snashall PD, Parker S, Ten Haave P, Simmons D, Noble MI. Use of an impedance meter for measuring airways responsiveness to histamine. Chest 1991; 99: 11831185.

17. Neild JE, Twort $\mathrm{CH}$, Chinn S, et al. The repeatability and validity of respiratory resistance measured by the forced oscillation technique. Respir Med 1989; 83: 111-118.

18. Van de Woestijne KP, Desager KN, Duiverman EJ, Marchal F. Recommendations for measurement of respiratory input impedance by means of the forced oscillation method. Eur Respir Rev 1994; 4: 235-237.

19. Morlion B, Knoop C, Paiva M, Estenne M. Internetbased home monitoring of pulmonary function after lung transplantation. Am J Respir Crit Care Med 2002; 165: 694697.

20. Finkelstein J, Cabrera MR, Hripcsak G. Internet-based home asthma telemonitoring: can patients handle the technology? Chest 2000; 117: 148-155.

21. Mazurek HK, Marchal F, Derelle J, Hatahet R, MoneretVautrin D, Monin P. Specificity and sensitivity of respiratory impedance in assessing reversibility of airway obstruction in children. Chest 1995; 107: 996-1002.

22. Delacourt C, Lorino H, Herve-Guillot M, Reinert P, Harf A, Housset B. Use of the forced oscillation technique to assess airway obstruction and reversibility in children. Am J Respir Crit Care Med 2000; 161: 730-736.

23. Nielsen KG, Bisgaard H. Discriminative capacity of bronchodilator response measured with three different lung function techniques in asthmatic and healthy children aged 2 to 5 years. Am J Respir Crit Care Med 2001; 164: 554-559.

24. Delacourt C, Lorino H, Fuhrman C, et al. Comparison of the forced oscillation technique and the interrupter technique for assessing airway obstruction and its reversibility in children. Am J Respir Crit Care Med 2001; 164: 965-972. 